\title{
Novel Microstrip Antenna Design
}

\author{
A. Hameed ${ }^{1}$, A. Oudah ${ }^{2}$ and Izzeldin I. Mohd ${ }^{3}$ \\ Faculty of Manufacturing Engineering ${ }^{1,2}$ \\ Faculty of Electrical \& Electronics Engineering ${ }^{3}$ \\ University Malaysia Pahang \\ Multicore.processor ${ }^{1}$,mark6ge@gmail.com²,izzeldin@ump.edu.my ${ }^{3}$
}

\begin{abstract}
A simple, small, compact, low cost, and practical antenna for $2.4 \mathrm{GHz}$ applications is proposed in this paper. A detailed investigation on miniaturized microstrip plana antenna design using a combination of proposed shorted patch and meandering method are presented. All design and simulations are done using Computer Simutlation Studio (CST) software. For accuracy reasons, verification has been done, all the destgns were fabricated, and measurements on return loss and impedance bandwodth were implemented by using RF Analyzer.

\section{Keywords: Antenna, microstrip, RF analyzer}

\section{Introduction}

Wireless feature implementations bave rapidly evolved over the past decades. Driven by this rapid progress, requirement for maller and cheaper antenna design becomes an integral factor in the deployment of wireless enabled products. It is evident that the wireless feature has become an important aspect in an introduction of any new products nowadays, regardless it being mobile or not. Manufacturers nowadays are pushing for a cheaper, smaller, simpler and more integrated way to implement wireless features [1]. One key element in wireless feature is the antepna design. Choosing the appropriate antenna design is crucial in order to optimize the wireless coverage and robustness of the transmission. The selection will also reflect to the material cost implementation simplicity and manufacturability of the product. The size of the antenna s an important factor. Small sized is typically desirable since it is generally cheaper and flexible to be used in small products. Nevertheless the limitation of antenna size is still bounded to its relation the wavelength of the transmission [1]. Thus reductions of antenna generally will have a negative impact to the performance. In most cases, trade-off needs to be done in order to get good balance between all of these factors [2]. Understanding in the standard requirements and a good knowledge on the variation of antennardesign will greatly aid this trading off process. It is well known that the microstrip antennas have been widely used in the past decade for wireless enabled devices. This is mainly due to its attractive characteristics such as small size, low profile, low cost and good repeatability, among others. Although microstrip antennas is by conventions are physically small, the requirement to further shrinkage in size is still highly desired [3].

In this article, we implementing a combination of the proposed patch shorting and meandering technique for antenna size reduction. Target resonance frequency is set to 2.4 GHz. The shorted patch method realized by the theory that when an antenna is operating in the lowest mode, a virtual short circuit forms through a plane centered between the two radiating edges [4]. This is achieved by implementing either a shorting wall, shorting plate or
\end{abstract}


shorting pin. The proposed method in this implementation is the Planar Inverted F-Antenna (PIFA). As for meandering technique, resonant frequency is lowered by lengthening surface current paths. This is achieved by introducing disruption on the resonant-length path causing the surface current to wonder according to a longer path. The proposed method in this implementation is the Meandered Line Antenna (MLA) and the Bowtie Antenna [4].

In ensuring this work is relevant to a practical implementation of a wireless module commonly used in the industry, a few limitations are set. The following describes these limitations and its reasoning:

- Microstrip-Fed Antenna: This feeding method is more practical especially for integrated antenna wireless module since RF circuit and antenna will be on the same side of the Printed Circuit Board (PCB).

- Low Cost PCB material: Due to economy of scale, FR4 PCB material is significantly cheaper than others. 2 layer FR4 PCB is chosen in this work.

- $\quad$ Full ground plane backed: To minimize internal interference from the product itself to the wireless transmission, a full grounded antenna structure is maintained.

- 2-D structure: To simplify manufacturability and repeatability of the antenna a 2D antenna structure is proposed. The antenna design and analysi in this work utilizes CST studio simulation software.

\section{Design methods}

A microstrip rectangular patch antenna is one the most basic antenna configuration used for practical application. A rectangular patch antenna can be represented as an array of two radiating narrow apertures, each of width, $W$ and heights, h, separated by a distance, L [5], typically half wavelength of the intended resonance frequency as illustrated in Figure 1. Because the dimensions of the patch are finite along the length and width, the fields at the edges of the pateh undergo fringing. Radiation will occur from this fringing field which extends the effective open circuit beyond the edge.

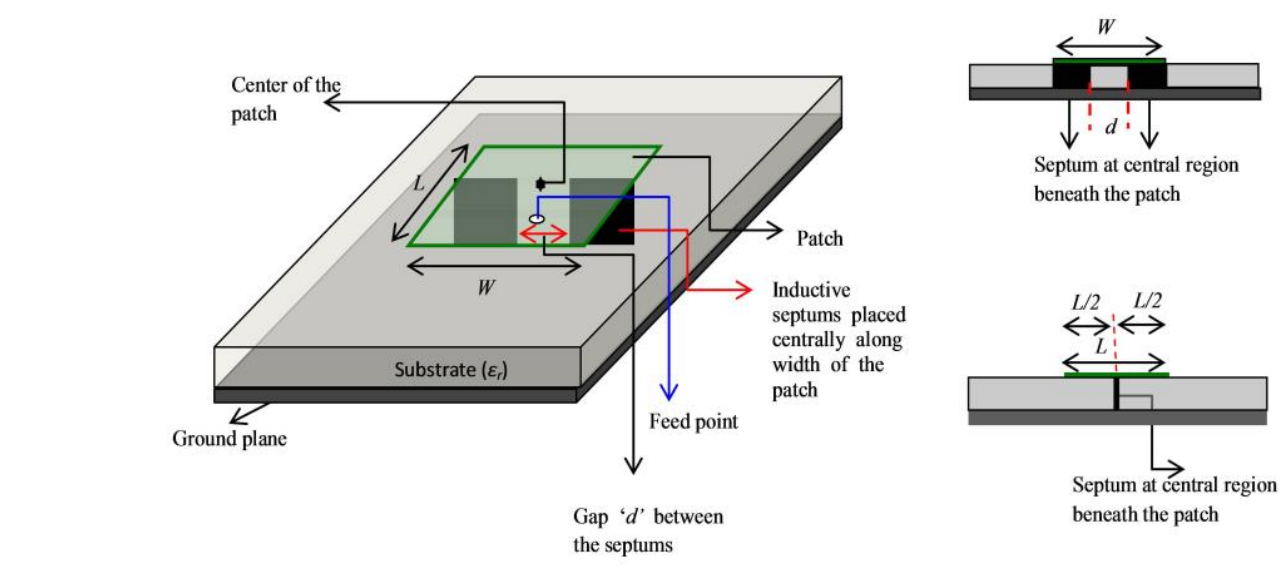

Figure 1. Antenna Structure

Due to the fringing field, the patch is electrically slightly larger than its actual physical length. This difference between electrical and physical length is dependent on the height and dielectric constant of the PCB. This deviation is given by formula below. 


$$
\Delta L=\frac{0.412(h)\left(\varepsilon_{\text {eff }}(\mathrm{W})+0.3\right)\left(\frac{W}{h}+0.26\right)}{\left(\varepsilon_{e f f}(W)-0.25\right)\left(\frac{W}{h}+0.8\right)}
$$

The effective dielectric constant, $\varepsilon_{\text {eff }}$ can be further reduced to:

$$
\varepsilon_{\text {eff }}=\frac{\varepsilon_{r}+1}{2}+\frac{\varepsilon_{r}-1}{2}\left[1+12 \frac{h}{W}\right]^{-\frac{1}{2}}
$$

$\varepsilon_{r}$ is the substrate dielectric constant.

Since the length of the patch has been extended by $\Delta \mathrm{L}$ on each side the effective length, $L_{\text {eff }}$ of the patch is now ( $\mathrm{L}=\lambda / 2$ for dominant $\mathrm{T}<010$ mode with no fringing).

$$
L_{e f f}=L+2 \Lambda L
$$

For the dominant TM010 mode, the resonant frequency, $f_{\text {res }}$ is dependent on the length of the antenna itself, which is written as:

$$
f_{\text {res }}=\frac{1}{2 L \frac{1}{L_{e f f}} \sqrt{\varepsilon_{\text {eff }}} \sqrt{\varepsilon_{0} \omega_{\theta}}}=\frac{c}{2 L \sqrt{\varepsilon_{\text {eff }}}}
$$

Where $\mu_{0}, \varepsilon_{\mathrm{o}}$, and $\mathrm{c}$ are the ree space permeability, permittivity and speed of light, respectively. This gives the equation of patch physical length in relation of resonant frequency. For a design of an efficient radiator, the following equation solves an optimized width, $W$ that provides good radiation efficiency, [5].

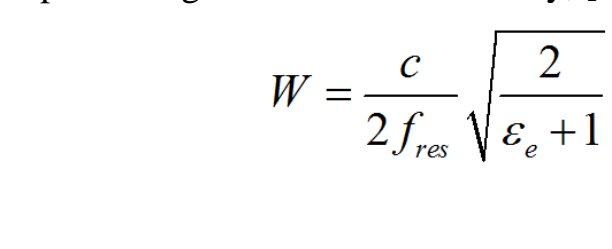

\section{Simulation, Analysis and Fabrication}

In this work, a conventional rectangular patch antenna with inset feed is designed by using transmission line model and simulation. This is aimed to be a benchmark for antenna miniaturization study in terms of size and radiation properties. Consistency of PCB parameters in simulation and material in fabrication is maintained for accurate comparison. The dimensions are realized in the theory $[5,6]$. By solving the equation discussed is section 2 , a conventional rectangular patch was designed as performance benchmark in this study. The target resonance frequency, $f_{\text {res }}$ is $2.45 \mathrm{GHz}$ with center frequency of $2.4 \mathrm{GHz}$ ISM band. Figure 2(a) illustrates the dimension of the conventional antenna. To confirm the actual performance of the designed and simulated antenna, the design is fabricated by using actual mass producer of PCB as in Figure 2(b). 


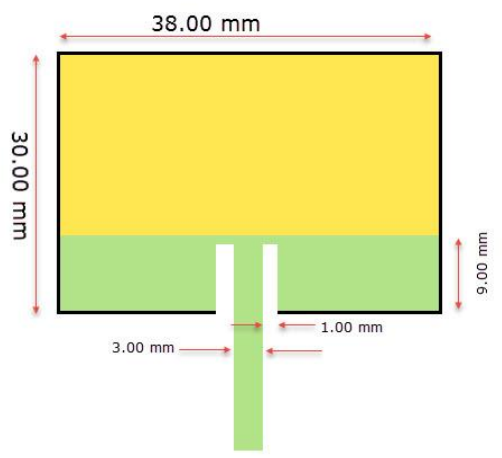

(a) (b)

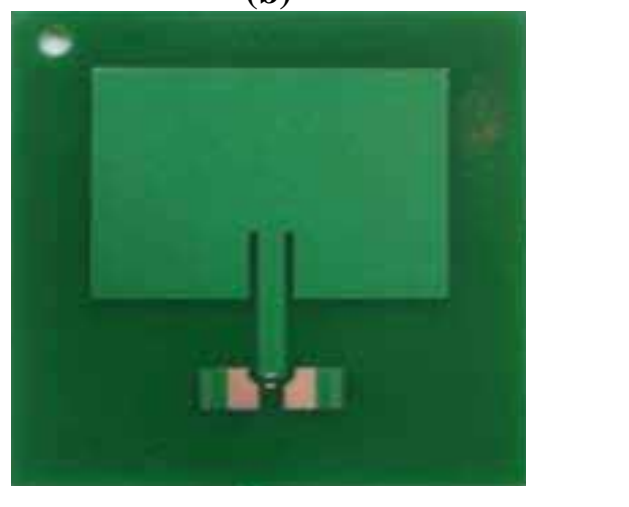

Figure 2. Proposed Antenna, (a) Measures (b) Fabrication

Table 1. Simulation Parameters

\begin{tabular}{|c|c|}
\hline Parameter & Value \\
\hline Size & $\frac{111 \mathrm{~mm} \mathrm{~mm}^{2}}{2.4} \mathrm{GHz}$ \\
\hline Resonance frequency & $2.14 \%$ \\
\hline 10dB bandwidth & $-35 \mathrm{~dB}$ \\
\hline Return loss & $2.4 \mathrm{dBi}$ \\
\hline Gain & $\mathrm{aBi}$ \\
\hline Directivity & $\mathrm{Gi}$ \\
\hline
\end{tabular}

\section{Results and Discussion}

A few miniaturized antenna designs were attempted in this work to observe the working principle of proposed shored patch and meandering. The first design is based on shorting wall concept where a series of vias are added in the middle of the patch where current is set maximum and voltage is zero. The quantity and positioning were tuned to optimize the size and reduce inductive effects. The size of antenna is half of conventional patch where $L \approx \lambda / 4$ as [7]. Figure 3 shows the resuts of surface analysis for the proposed antenna.

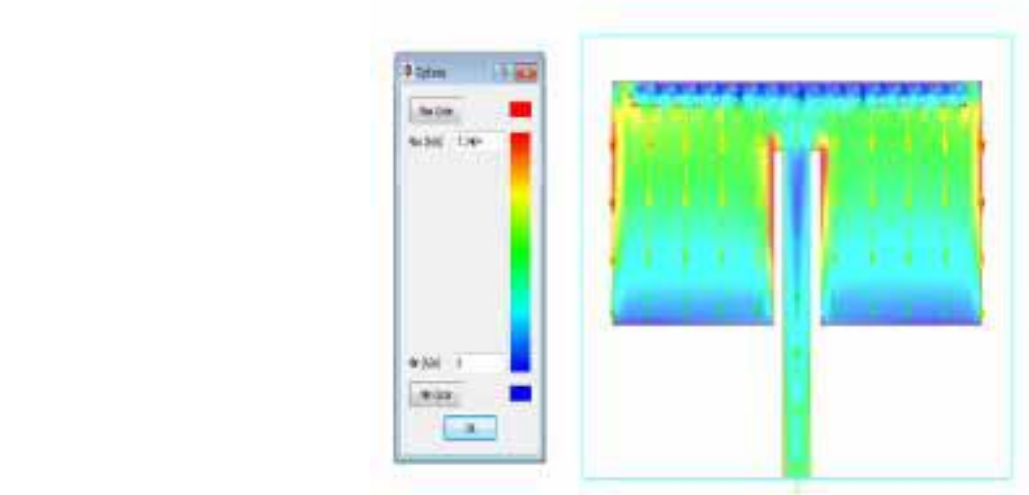

Figure 3. Schematic of the LNA to be Improved [3]

As shown in Figure 4, in this design we obtained return loss of $-30.4 \mathrm{~dB}$ and an accurate desired resonance frequency at $2.45 \mathrm{GHz}$ is achieved. This is true as long as impedance of the 
pin shorting remains low. With high impedance shorting path the antenna will detune and causes the resonance frequency to be shifted as [7]. It is also observed that the impedance bandwidth of the antenna at $10 \mathrm{~dB}$ is $1.7 \%$ which is a little reduced compared to a full patch. This reduction also causes the radiation pattern to behave differently compared to a full patch antenna. As seen in Figure 6, the radiation spreads equally above the ground plane. This result a lowers the directivity compared to a full patch antenna. The simulated directivity of this antenna is $4.61 \mathrm{~dB}$. The efficiency remains the same at $45 \%$.

(a)

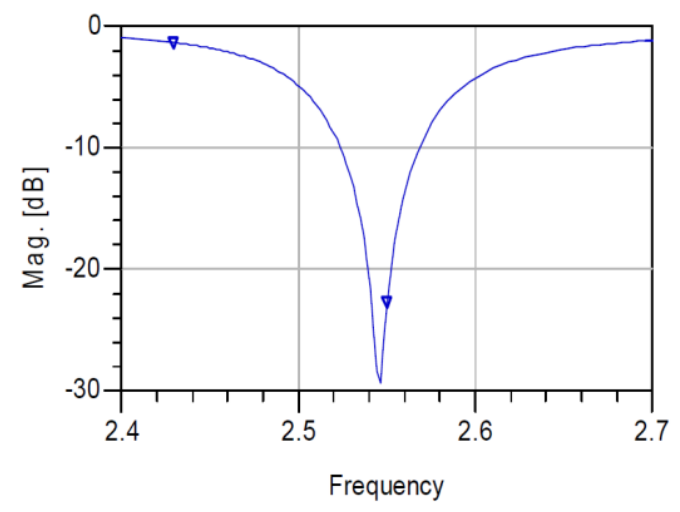

(b)

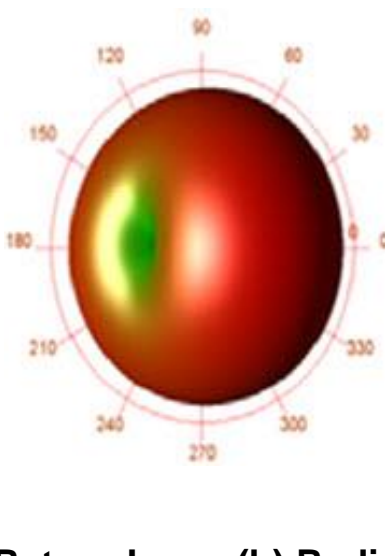

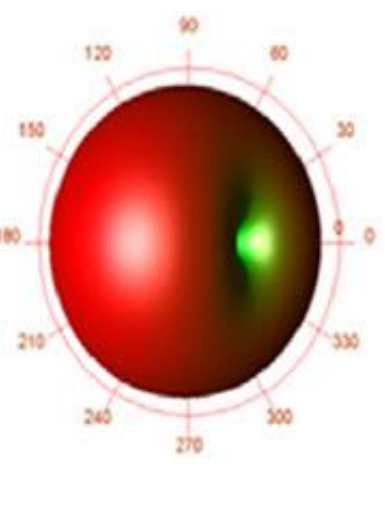

Figure 4. Simulation Results (a) Return Loss, (b) Radiation Pattern

\section{Conclusions}

Novel, compact and low cost suspended plate antenna miniaturization design for $2.4 \mathrm{GHz}$ application has been presented. It is observed that significant size reduction from a conventional rectangular patch antenna can be achieved by implementing these proposed techniques. For this, the radiation properties such as impedance band width, return loss, gain, बirectivity, efficiency, radiation pattern and surface current was discussed and anayzed. Improvements were also made based on best efforts to minimize the degradation.

\section{References}

[1] S. Choi, D. Kwak, H. Lee and K. Kwak, "Design of a Dual-Band pi-Shaped Microstrip Patch Antenna with a Shorting Pin for 5.2/5.8GHz WLAN Systems", Microwave and Optical Technology Letters, vol. 52, (2010), pp. 825-827.

[2] A. P. Dhande and V. Ambhore, "An Overview on Properties, Parameter Consideration And Design of Meandering Antenna", International Journal of Smart Sensors and Ad Hoc Networks (IJSSAN), (2012), 5962.

[3] D. Fang, Antenna Theory and Microstrip Antenna”, CRC Press, (2010).

[4] M. Sarkar and S. Chowdhury, "A New Compact Microstrip Patch Antenna", Microwave and Optical Technology Letters, vol. 47, no. 4, (2005), 379-381.

[5] A. K. Skrivervik, J. F. Zurcher, O. Staub and J. Mosig, "PCS antenna design: the challenge of miniaturization", IEEE Antennas and Propagation Magazine, vol. 43, no. 4, (2001), 12-27.

[6] A. Vasylchenko, Y. Schols, W. D. Raedt and G. A. Vandenbosch, "A Benchmarking of Six Software Packages for Full-Wave Analysis of Microstrip Antennas", (2008).

[7] Y. S. Vandenbosch, "Separation of horizontal and vertical dependencies in a surface/volume integral equation approach to model quasi 3-D structures in multilayered media", IEEE Trans. Antennas Propagation, vol. 55, no. 32, (2007). 
International Journal of Smart Home

Vol. 8, No. 6 (2014)

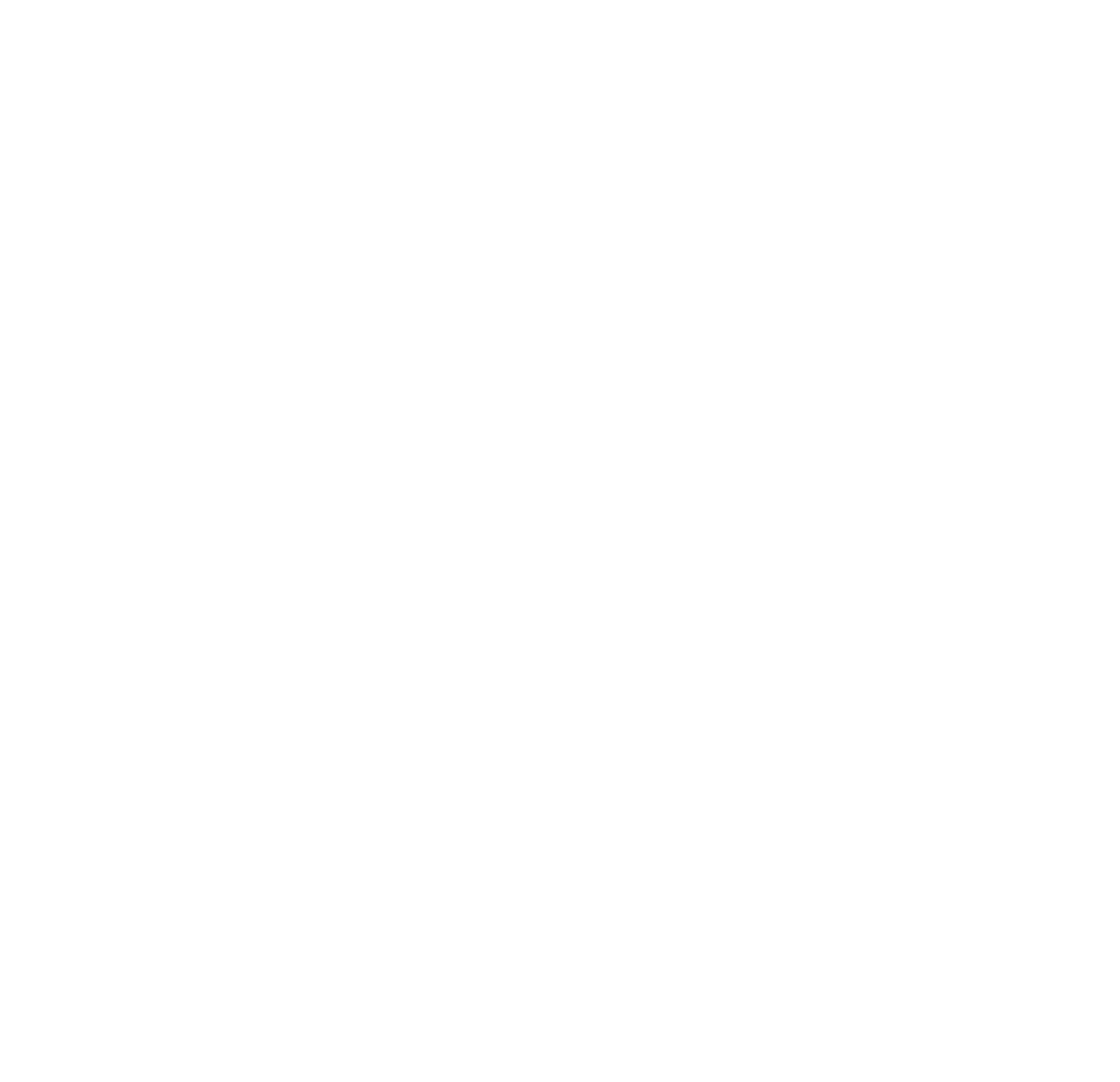

